\title{
The Association between End-of-Life Care and the Time Interval between Provision of a Do-Not-Resuscitate Consent and Death in Cancer Patients in Korea
}

\author{
Sun Kyung Baek, MD, PhD ${ }^{1}$ \\ Hye Jung Chang, MD2 \\ Ja Min Byun, $\mathrm{MD}^{3}$ \\ Jae Joon Han, MD, $P h D^{3}$ \\ Dae Seog Heo, MD, PhD
}

${ }^{1}$ Department of Internal Medicine, Kyung Hee University Hospital, Seoul, ${ }^{2}$ Department of Internal Medicine, Kyung Hee University Hospital at Gangdong, Seoul, ${ }^{3}$ Department of Internal Medicine, Seoul National University Hospital, Seoul, Korea

Correspondence: Dae Seog Heo, MD, PhD Department of Internal Medicine,

Seoul National University Hospital, 101 Daehak-ro, Jongno-gu, Seoul 03080, Korea Tel: $82-2-2072-2857$

Fax: 82-2-742-6689

Email: heo1013@snu.ac.kr

Received February 17, 2016

Accepted August 11, 2016

Published Online September 1, 2016

\begin{abstract}
Purpose
We explored the relationship between the use of each medical intervention and the length of time between do-not-resuscitate (DNR) consent and death in Korea.
\end{abstract}

\section{Materials and Methods}

A total of 295 terminal cancer patients participated in this retrospective study. Invasive interventions (e.g., cardiopulmonary resuscitation, intubation, and hemodialysis), less invasive interventions (e.g., transfusion, antibiotic use, inotropic use, and laboratory tests), and the time interval between the DNR order and death were evaluated. The subjects were divided into three groups based on the amount of time between DNR consent and death (G1, time interval $\leq 1$ day; G2, time interval $>1$ day to $\leq 3$ days; and G3, time interval $>3$ days).

\section{Results}

In general, there were fewer transfusions and laboratory tests near death. Invasive interventions tended to be implemented only in the G1 group. There was also less inotrope use and fewer laboratory tests in the G3 group than G1 and G2. Moreover, the G3 group received fewer less invasive interventions than those in $\mathrm{G} 1$ (odds ratio [OR], 0.16; 95\% confidence interval [Cl], 0.03 to $0.84 ; 3$ days before death, and $\mathrm{OR}, 0.16 ; 95 \% \mathrm{Cl}, 0.04$ to 0.59 ; the day before death). The frequency of less invasive interventions both 1 and 3 days before death was significantly lower for the G3 group than the G1 ( $p \leq 0.001)$ and G2 group compared to $G 1$ ( $p=0.001)$.

\section{Conclusion}

Earlier attainment of DNR permission was associated with reduced use of medical intervention. Thus, physicians should discuss death with terminal cancer patients at the earliest practical time to prevent unnecessary and uncomfortable procedures and reduce health care costs.

\section{Introduction}

The purpose of the end-of-life (EOL) discussion is to allow dying patients to die peacefully without undergoing aggressive interventions before death. In Korea, do-not-resuscitate (DNR) is an order to withhold cardiopulmonary resuscitation (CPR) or advanced cardiac life support in respect of the wishes of a patient in case they were to stop breathing or their heart were to stop. A DNR directive must be explained
Key words

Neoplasms, Resuscitation orders, Terminal care to the patients or the surrogate decision maker during EOL care. Indeed, the DNR directive involves ethical, legal, medical, and economical considerations, and many studies have shown that DNR consent has wide cultural differences [1,2].

In Korea, cultural values and social norms preclude active discussions regarding death. Thus, physicians, family members and patients experience considerable difficulties when communicating with one another about the subject [3]. Equally importantly, the Korean legal system has yet to acknowledge the legal authority of DNR documentation; 
therefore, EOL care planning is only conducted when death is imminent $[4,5]$. However, late DNR consent is associated with increased hospital cost [6] and patients receiving unnecessary medical intervention to sustain life [7].

Many experts have recommended that EOL care discussions be initiated while the patient can still actively participate in the discussion $[8,9]$. However, to the best of our knowledge, there is a lack of data regarding the association between each EOL intervention and the time interval between DNR consent and death, especially in regions such as Korea, where proxy decision-making and imminent DNR consent near death are a common social practice. Therefore, we investigated decision making and current medical practices relating to EOL care of terminal cancer patients in Korea. We also evaluated the association between EOL care and the time interval between provision of DNR consent and death.

\section{Materials and Methods}

We designed a retrospective cohort study composed of patients who had been admitted into expire rooms (designated as rooms for peaceful death), which require DNR consent to occupy, and who died between July 2006 and June 2009 in Seoul National University Hospital (SNUH), a tertiary referral hospital. In $\mathrm{SNUH}$, most patients are referred from primary care physicians or secondary hospitals and receive active chemotherapy and cancer surgery. There is no inpatient hospice unit, and patients who do not want to transfer to a regional hospice center or secondary hospital for hospice care die at SNUH. Patients were required to meet these criteria: admission and death in an expire room, provided DNR consent, being over 18 years of age, diagnosed with cancer and having medical records showing consent and the time interval between DNR consent and death. This study was approved by the Institutional Review Board of SNUH (H-1602-047-739).

Among the 322 patients who expired in an expire room during the study period, 321 died due to terminal cancer. Information about the time interval between DNR consent and death was available for 295 patients of these 321 patients. Medical records and DNR consent were reviewed. Clinical data including age, sex, route of admission, type of cancer, whether verbal or written DNR consent was provided, person(s) who consented DNR (in person or by proxy), and the time interval between DNR consent and death were obtained. DNR consent was defined as the patient having a preprinted DNR form or recorded verbal communication on a medical note. EOL care consisted of invasive medical inter- vention (e.g., CPR, intubation, and hemodialysis) within 7 days of death and less aggressive medical intervention (e.g., transfusion, antibiotic use, inotropic use, and laboratory test) within 7 days of death.

The subjects were divided into three groups (G1, G2, and G3) according to the time interval between DNR consent and death $(\mathrm{G} 1,0<$ time interval $\leq 1$ day; $\mathrm{G} 2,1$ day $<$ time interval $\leq 3$ days; and G3, 3 days $<$ time interval). For each individual, implementation of each less aggressive intervention was given a score of 1 (implementation) and 0 (no implementation). All scores were summed to yield the "less aggressive intervention score," which ranged from 0 to 4.

\section{Statistical analysis}

All data are presented as numbers (\%). Implementation of less aggressive interventions within 1,3 , and 7 days of death was compared using the chi-squared test. The subject characteristics were compared among the three groups using chisquared tests for categorical variables. Multiple logistic regression analyses for implementation of any less aggressive intervention were used to generate odds ratios (ORs) (95\% confidence intervals [95\% CIs]) relative to the tertile of the time interval between DNR consent and death. Analysis of covariance (ANCOVA) was used to compare the less aggressive intervention score according to the tertiles. All statistical analyses were performed using SPSS ver. 18.0 (SPSS Inc., Chicago, IL) and a $\mathrm{p} \leq 0.05$ was considered statistically significant.

\section{Results}

\section{Patient demographic characteristics}

Patient characteristics are shown in Table 1. There were 204 patients $(69.2 \%)<65$ years and 160 males $(54.2 \%)$. Overall, 249 patients $(84.4 \%)$ were admitted via the outpatient department and the others were admitted via the emergency room. The number of cancer patients with solid tumors was $263(89.2 \%)$, while the rest had hematologic malignancies. In total, 246 patients $(83.4 \%)$ provided a written DNR consent and $49(16.6 \%)$ provided verbal DNR consent. DNR consent was made between the physician and family, without involving the patient. Persons who consented to DNR were primarily the patients' spouse $(\mathrm{n}=142,48.1 \%)$ and offspring/ parents $(n=118,40.0 \%)$, followed by a relative or someone with an uncertain family relation $(\mathrm{n}=35,11.9 \%)$.

DNR directives were enacted at a median of 1.76 days (range, 0.0 to 100.48 days) before death. A DNR discussion 
Table 1. Baseline characteristics

\begin{tabular}{|c|c|}
\hline Variable & No. $(\%)(n=295)$ \\
\hline Age, median (range, yr) & $50(20-89)$ \\
\hline$<65$ & $204(69.2)$ \\
\hline$\geq 65$ & $91(30.8)$ \\
\hline \multicolumn{2}{|l|}{ Sex } \\
\hline Female & $135(45.8)$ \\
\hline Male & $160(54.2)$ \\
\hline \multicolumn{2}{|l|}{ Route of admission } \\
\hline Emergency room & $46(15.6)$ \\
\hline Out-patient clinic & $249(84.4)$ \\
\hline \multicolumn{2}{|l|}{ Type of cancer } \\
\hline Solid tumor & $263(89.2)$ \\
\hline Hematologic malignancy & $32(10.8)$ \\
\hline \multicolumn{2}{|l|}{ Form of DNR consent } \\
\hline Verbal consent & $49(16.6)$ \\
\hline Preprinted written form & $246(83.4)$ \\
\hline \multicolumn{2}{|l|}{ Person who consents to DNR } \\
\hline Patient & 0 \\
\hline Family & $295(100)$ \\
\hline Spouse & $142(48.1)$ \\
\hline Parent(s)/Offspring & $118(40.0)$ \\
\hline Other relative(s) & $35(11.9)$ \\
\hline Aggressive interventions & $7(2.4)$ \\
\hline CPR & $6(2.0)$ \\
\hline Intubation & $3(1.0)$ \\
\hline Hemodialysis & $1(0.3)$ \\
\hline Less aggressive interventions & $290(98.3)$ \\
\hline Transfusion & $133(45.1)$ \\
\hline Antibiotic use & $240(81.4)$ \\
\hline Inotropic use & $110(37.3)$ \\
\hline Laboratory test & $275(93.2)$ \\
\hline \multicolumn{2}{|l|}{$\begin{array}{l}\text { Time interval between } \\
\text { DNR consent and death }\end{array}$} \\
\hline Median (range, day) & $1.76(0.0-100.48)$ \\
\hline Group $1(\mathrm{n}=83)$ & $0.00-1.00$ \\
\hline Group 2 (n=108) & $1.00-3.00$ \\
\hline Group $3(n=104)$ & $3.00-100.48$ \\
\hline
\end{tabular}

DNR, do-not-resuscitate; CPR, cardiopulmonary resuscitation. was made within 3 days of death for $65 \%$ of the patients. Subjects were divided into three groups $(\mathrm{G})$ based on the time interval between DNR consent and death: G1 (time interval $\leq 1$ day, $\mathrm{n}=83$ ), $\mathrm{G} 2$ (time interval $>1$ day to $\leq 3$ days, $\mathrm{n}=108$ ), and G3 (time interval $>3$ days, $\mathrm{n}=104$ ) (Table 1 ).

For invasive interventions, CPR was performed on six patients, intubation on three patients and hemodialysis on one patient within the 7 days before death (Table 1). Table 2 shows the other (less aggressive) interventions performed within 1,3, and 7 days of death. The frequency of transfusions and laboratory tests were significantly lower near death, when the majority of the participants had provided DNR consent (Table 2). However, use of antibiotics and inotropes did not decrease.

\section{Patient characteristics according to groups of the time interval between DNR consent and death}

Age, sex, diagnosis, verbal or written DNR consent, and person consenting DNR were similar among the three groups (Table 3). However, group G1 included more patients admitted via the emergency room $(p<0.001)$ (Table 3$)$ than the other two groups.

Invasive interventions, e.g., CPR, intubation and hemodialysis, were only implemented in group G1 (Table 4). Among other less aggressive interventions, use of inotropes was significantly lower in group G2 than groups G1 (Table 4). Group G3 also had fewer laboratory tests than group G2 (Table 4). There was no significant difference in the number of transfusions or antibiotic use among groups G1-G3.

\section{Association between medical interventions and the time between DNR consent and death}

Table 5 shows the association between medical interventions and the time interval between DNR consent and death after adjustment for various confounders. Three days before death, all participants in group G3 had provided DNR consent and they received fewer less aggressive interventions

Table 2. Less aggressive interventions performed during the 7 days before death

\begin{tabular}{lccrrc} 
& Total & Within 7 days & Within 3 days & Within 1 day & p-value \\
Transfusion & $133(45.1)$ & $133(45.1)$ & $94(31.9)$ & $59(20.0)$ & $<0.001^{*}$ \\
Antibiotics use & $240(81.4)$ & $239(81.0)$ & $228(77.3)$ & $222(75.3)$ & 0.232 \\
Inotropes use & $110(37.3)$ & $107(36.3)$ & $106(35.9)$ & $103(34.9)$ & 0.938 \\
Laboratory tests & $275(93.2)$ & $275(93.2)$ & $238(80.7)$ & $204(69.2)$ & $<0.001^{*}$ \\
\hline
\end{tabular}

Values are presented as number $(\%)$. *Significant results $(\mathrm{p} \leq 0.05)$. 
Table 3. Baseline characteristics of patients divided into G1/2/3 based on the time interval between DNR consent and death

\begin{tabular}{|c|c|c|c|c|c|}
\hline Variable & Total $(\mathrm{n}=295)$ & G1 $(n=83)$ & G2 $(n=108)$ & G3 $(n=104)$ & p-value \\
\hline \multicolumn{6}{|l|}{ Age (yr) } \\
\hline$<65$ & $204(69.2)$ & $59(71.1)$ & $74(68.5)$ & $71(68.3)$ & 0.903 \\
\hline$\geq 65$ & $91(30.8)$ & $24(28.9)$ & $34(31.5)$ & $33(31.7)$ & \\
\hline \multicolumn{6}{|l|}{ Sex } \\
\hline Female & $135(45.8)$ & $50(60.2)$ & $56(51.9)$ & $50(48.1)$ & 0.432 \\
\hline Male & $160(54.2)$ & $33(39.8)$ & $52(48.1)$ & $54(51.9)$ & \\
\hline \multicolumn{6}{|l|}{ Route of admission } \\
\hline Emergency room & $46(15.6)$ & $22(26.5)$ & $18(16.7)$ & $6(5.8)$ & $0.000^{*}$ \\
\hline Out-patient clinic & $249(84.4)$ & $61(73.5)$ & $90(83.3)$ & $98(94.2)$ & \\
\hline \multicolumn{6}{|l|}{ Type of cancer } \\
\hline Solid tumor & $263(89.2)$ & $70(84.3)$ & 99 (91.7) & $94(90.4)$ & 0.239 \\
\hline Hematologic malignancy & $32(10.8)$ & $13(15.7)$ & $9(8.3)$ & $10(9.6)$ & \\
\hline \multicolumn{6}{|l|}{ Form of DNR consent } \\
\hline Verbal consent & $49(16.6)$ & $17(20.5)$ & $20(18.5)$ & $12(11.5)$ & 0.211 \\
\hline Preprinted written consent & $246(83.4)$ & $66(79.5)$ & $88(81.5)$ & $92(88.5)$ & \\
\hline \multicolumn{6}{|l|}{ Person who consented DNR } \\
\hline Spouse & $141(47.8)$ & $33(39.8)$ & $53(49.1)$ & $55(52.9)$ & 0.155 \\
\hline Offspring/Parents & $118(40.0)$ & $34(41.0)$ & $44(40.7)$ & $40(38.5)$ & \\
\hline Relatives & $36(12.2)$ & $16(19.3)$ & $11(10.2)$ & $9(8.7)$ & \\
\hline
\end{tabular}

Values are presented as number (\%). G1 (group 1), time interval $\leq 1$ day; G2 (group 2), 1 day $<$ time interval $\leq 3$ days; G3 (group 3), 3 days < time interval; DNR, do-not-resuscitate. *Significant results $(\mathrm{p} \leq 0.05)$.

Table 4. End-of-life care according to tertiles, based on the time interval between the DNR consent and death

\begin{tabular}{|c|c|c|c|c|c|}
\hline Variable & Total $(n=295)$ & G1 $(n=83)$ & G2 $(n=108)$ & G3 (n=104) & p-value ${ }^{a)}$ \\
\hline Aggressive intervention & $7(2.4)$ & $7(8.4)$ & 0 & 0 & $0.000^{*}$ \\
\hline CPR & $6(2.0)$ & $6(7.2)$ & 0 & 0 & $0.000^{*}$ \\
\hline Intubation & $3(1.0)$ & $3(3.6)$ & 0 & 0 & $0.021^{*}$ \\
\hline Hemodialysis & $1(0.3)$ & $1(1.2)$ & 0 & 0 & 0.278 \\
\hline Other intervention & $290(98.3)$ & $83(100)$ & $107(99.1)$ & $100(96.2)$ & 0.095 \\
\hline Transfusion & $133(45.1)$ & $39(47.0)$ & $49(45.4)$ & $45(43.3)$ & 0.877 \\
\hline Antibiotic use & $240(81.4)$ & $65(78.3)$ & $89(82.4)$ & $86(82.7)$ & 0.702 \\
\hline Inotropic use & $110(37.3)$ & $46(55.4)$ & $36(33.3)^{b)}$ & $28(26.9)$ & $<0.001^{*}$ \\
\hline Laboratory tests & $275(93.2)$ & $78(94.0)$ & $105(97.2)$ & $92(88.5)^{c)}$ & $0.038^{*}$ \\
\hline
\end{tabular}

Values are presented as number (\%). DNR, do-not-resuscitate; G1 (group 1), time interval $\leq 1$ day; G2 (group 2), 1 day < time interval $\leq 3$ days; G3 (group 3), 3 days $<$ time interval; CPR, cardiopulmonary resuscitation. *Significant results ( $\mathrm{p} \leq 0.05$ ). ${ }^{a} \mathrm{p}$-value by chi-squared test, ${ }^{b}$ p-value was statistically significant $(\alpha<0.016)$ after Bonferroni correction for multiple testing $(\alpha=0.05 / 3)$ vs. G1 by chi-squared test, ${ }^{c}$ p-value was statistically significant $(\alpha<0.016)$ after Bonferroni correction for multiple testing $(\alpha=0.05 / 3)$ vs. G2 by chi-squared test.

than group G1, in which none of the members had provided DNR consent (OR, 0.12; 95\% CI, 0.01 to 1.03; $\mathrm{p}$ for trend= $0.017)$. On the day before death, patients in $\mathrm{G} 3$ received fewer less aggressive interventions than those in group G1 (OR, $0.14 ; 95 \% \mathrm{CI}, 0.03$ to 0.66 ; $\mathrm{p}$ for trend=0.005). Fig. 1 shows the less invasive intervention score according to amounts based on the time interval between DNR consent and death. Seven days before death, there were no significant differences in the number of less invasive interventions among G1, G2, and G3. The less invasive intervention score three days and one day before death was significantly lower for group G3 than groups G1 and G2. 
Table 5. Associations between groups based on the time interval between the DNR consent and death (hour) and less aggressive interventions

\begin{tabular}{lcccccc} 
& \multicolumn{3}{c}{ Odds ratio ${ }^{\mathrm{a}}(\mathbf{9 5} \%$ confidence interval) } \\
\cline { 2 - 7 } Variable & $\begin{array}{c}\text { Within } 5 \text { days } \\
\text { of death }\end{array}$ & $\begin{array}{c}\mathrm{p} \text { for } \\
\text { trend }\end{array}$ & $\begin{array}{c}\text { Within } 3 \text { days } \\
\text { of death }\end{array}$ & $\begin{array}{c}\mathrm{p} \text { for } \\
\text { trend }\end{array}$ & $\begin{array}{c}\text { Within } 1 \text { day } \\
\text { of death }\end{array}$ & $\begin{array}{c}\mathrm{p} \text { for } \\
\text { trend }\end{array}$ \\
G1 & Reference & 0.646 & Reference & $0.017^{*}$ & Reference & $0.005^{*}$ \\
G2 & NA & - & $0.32(0.03-3.04)$ & - & $0.29(0.06-1.44)$ & - \\
G3 & NA & - & $0.12(0.01-1.03)$ & - & $0.14^{*}(0.03-0.66)$ \\
\hline
\end{tabular}

DNR, do-not-resuscitate; G1 (group 1), time interval $\leq 1$ day; G2 (group 2), 1 day < time interval $\leq 3$ days; G3 (group 3), 3 days $<$ time interval. *Significant results $(\mathrm{p} \leq 0.05)$. a)Adjusted for age, sex, route of admission, type of cancer, form of DNR consent, person(s) who provided DNR consent, and aggressive intervention.

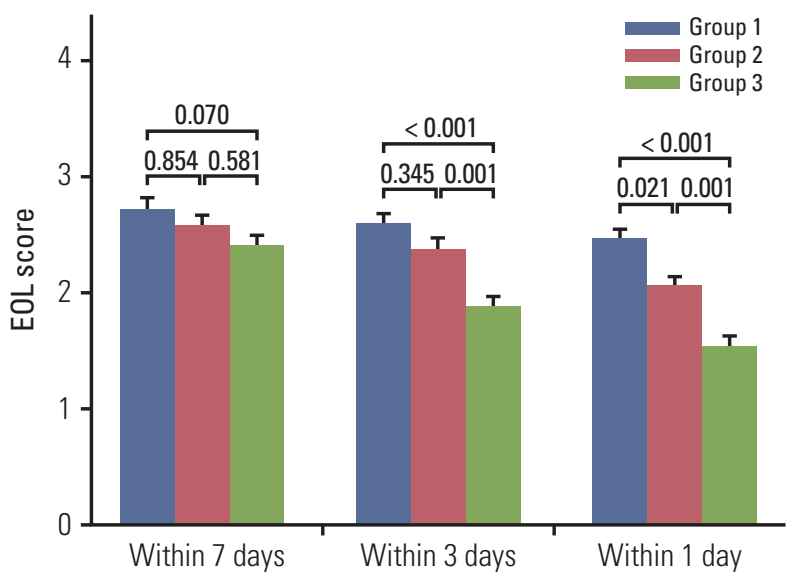

Fig. 1. Less aggressive intervention score (e.g., transfusion, antibiotic use, inotropic use, and laboratory tests) according to group (based on time interval between the DNR consent and death). DNR, do-not-resuscitate; EOL, end-of-life.

\section{Discussion}

Early EOL care discussions and planning could help patients meet death with peace and dignity. Previous data also indicate that such discussions lead to reduced medical costs and better allocation of medical resources by decreasing unnecessary and expensive invasive procedures $[6,7]$. Our results were consistent with these previous reports, and showed that those with earlier DNR consent received fewer aggressive interventions (CPR, intubation, and hemodialysis) and fewer less aggressive interventions, especially inotrope use and laboratory tests. Between $12 \%$ and $87 \%$ of patient groups provide DNR consent in Korea $[4,5,10]$. The median time from DNR consent to death in this study was 1.76 days, which is shorter than what has been reported in previous studies. This study was conducted at a tertiary hospital in which patients tend to receive more active anti-cancer therapy. At this facility, $12 \%$ of all cancer patients provide DNR consent, and the hospital does not have an inpatient hospice [10]. In general, patients at this facility have little time to discuss the DNR directive before death, and the surrogate(s) have little time to make important EOL care decisions [11].

In this study, fewer transfusions and laboratory tests were performed as the day of death approached. However, use of antibiotics and inotropes did not change, despite initiation of the DNR protocol. According to previous reports in Korea, $68 \%$ of the general population and $71 \%$ of medical professionals agree that there are ethical and legal differences between withdrawal of treatment and withholding life-sustaining treatment [12], with withholding being acceptable, but withdrawing unacceptable [13]. Our data demonstrate that these ideals were practiced in this setting. For example, if a patient had an infection or hypotension and had already begun to receive antibiotics or inotropes, the physician and/or family might think that withdrawal of those drugs was unethical. However, they may not consider it to be unethical to withhold laboratory tests or transfusions. In turn, these ethical ideals would decrease the frequency of one-time tests and interventions while maintaining regimens that last several days or weeks. Indeed, in our study, the use of antibiotics was not related to the time interval between DNR consent and death, and over $80 \%$ of patients were receiving antibiotics. However, those who consented to DNR at least several days before death exhibited less inotrope use and had fewer laboratory tests ordered than those who consented approximately one day before death (i.e., median of 1.76 days between DNR consent and death).

As mentioned in previous studies [4,5,14], DNR discus- 
sions and proxy decision-making of DNR consent is emotional, stressful and overwhelming for terminal patients and their families. In Asian cultures, making a decision to withhold or withdraw life-sustaining treatment from a close family member may feel unfilial or unaffectionate. Indeed, family caregivers tend to have a significantly more aggressive attitude toward EOL care for their ill family members than the patients' own stated preferences [15]. Life-sustaining medical interventions can prolong, albeit to a limited extent, the lives of cancer patients near death. While this can be very meaningful and valuable time for families, patients may feel a prolongation of suffering and hope for discontinuation of futile interventions.

The issues regarding EOL decisions for patients who are terminally ill reflect the different cultural backgrounds between Western and Eastern countries. In the United States and many European countries, there is a concept of autonomy and the best interest of the patients in decision making $[16,17]$. However, Korea, China, and Japan have a family-oriented Confucian culture, and many patients prefer to also consider the best of interest of all family members [18]. Although patients' autonomy in decision making is important, patients also wish to discuss EOL care with their families. Therefore, family members need to play a large role in the EOL discussion. Earlier discussions of EOL care can help mediate different perspectives among patients and family members regarding life prolongation. Accordingly, physicians should broach the subject of death with cancer patients and family members in advance and help them discuss their EOL care with family members.

It should be noted that there are several limitations to our study. First, because our study was a single-institution study, these results may not reflect typical national practices in Korea. Indeed, there are no large databases and DNR order styles vary drastically among hospitals; therefore, future work should investigate different practices regarding DNR consent from throughout the country. Second, the median time from DNR consent to death in this study was very short compared to other investigations, which again suggests that these data do not accurately represent Korean practices nationwide. However, data from this hospital can still be used to investigate the time interval between DNR consent and death and EOL care in cancer patients, as well as the decision-making course of EOL care near death. Finally, we did not include quality-of-life data. Nevertheless, this study is the first to assess the association between each EOL care intervention and the time interval between DNR consent and death.

\section{Conclusion}

In summary, the DNR decision by proxy was typically performed within the last days of the patients' life in a tertiary referral hospital in Korea. Earlier DNR permission (i.e., at least several days before death) allowed patients to receive fewer aggressive interventions, including CPR, as well as less aggressive interventions in the days before death. Therefore, to spare patients from unnecessary, potentially uncomfortable procedures and reduce medical costs, physicians should disclose terminal prognoses to cancer patients in advance and include patients in EOL care discussions.

\section{Conflicts of Interest}

Conflict of interest relevant to this article was not reported.

\section{Acknowledgments}

This study was supported by a grant from the Innovative Research Institute for Cell Therapy, Republic of Korea (A062260).

\section{References}

1. Jonsson PV, McNamee M, Campion EW. The 'Do not resuscitate' order: a profile of its changing use. Arch Intern Med. 1988;148:2373-5.

2. Rabkin MT, Gillerman G, Rice NR. Orders not to resuscitate. N Engl J Med. 1976;295:364-6.

3. Oh DY, Kim JE, Lee CH, Lim JS, Jung KH, Heo DS, et al. Dis- crepancies among patients, family members, and physicians in Korea in terms of values regarding the withholding of treatment from patients with terminal malignancies. Cancer. 2004;100:1961-6.

4. Kim DY, Lee KE, Nam EM, Lee HR, Lee KW, Kim JH, et al. Do-not-resuscitate orders for terminal patients with cancer in 
teaching hospitals of Korea. J Palliat Med. 2007;10:1153-8.

5. Oh DY, Kim JH, Kim DW, Im SA, Kim TY, Heo DS, et al. CPR or DNR? End-of-life decision in Korean cancer patients: a single center's experience. Support Care Cancer. 2006;14:103-8.

6. Celso BG, Meenrajan S. The triad that matters: palliative medicine, code status, and health care costs. Am J Hosp Palliat Care. 2010;27:398-401.

7. Becker G, Sarhatlic R, Olschewski M, Xander C, Momm F, Blum HE. End-of-life care in hospital: current practice and potentials for improvement. J Pain Symptom Manage. 2007; 33:711-9.

8. Kasper DL, Fauci AS, Hauser SL, Longo DL, Jameson JL, Loscalzo J. Harrison's principles of internal medicine. 19th ed. New York: McGraw Hill Education Medical; 2015.

9. National Institute for Health and Care Excellence. Improving supportive and palliative care for adults with cancer. London: National Institute for Health and Care Excellence; 2004.

10. Keam B, Oh DY, Lee SH, Kim DW, Kim MR, Im SA, et al. Aggressiveness of cancer-care near the end-of-life in Korea. Jpn J Clin Oncol. 2008;38:381-6.

11. Lee JK, Keam B, An AR, Kim TM, Lee SH, Kim DW, et al. Surrogate decision-making in Korean patients with advanced cancer: a longitudinal study. Support Care Cancer. 2013;21:183-90.

12. National Evidence-based Healthcare Collaboration Agency.
Social consensus for withdrawal of futile life-sustaining treatment. Seoul: National Evidence-based Healthcare Collaboration Agency; 2009.

13. Heo DS. Life-sustaining medical treatment for terminal patients in Korea. J Korean Med Sci. 2013;28:1-3.

14. Gu X, Chen M, Liu M, Zhang Z, Cheng W. End-of-life decision-making of terminally ill cancer patients in a tertiary cancer center in Shanghai, China. Support Care Cancer. 2016;24: 2209-15.

15. Tang ST, Liu TW, Lai MS, Liu LN, Chen CH. Concordance of preferences for end-of-life care between terminally ill cancer patients and their family caregivers in Taiwan. J Pain Symptom Manage. 2005;30:510-8.

16. Andorno R, Biller-Andorno N, Brauer S. Advance health care directives: towards a coordinated European policy? Eur J Health Law. 2009;16:207-27.

17. Teno JM, Gruneir A, Schwartz Z, Nanda A, Wetle T. Association between advance directives and quality of end-of-life care: a national study. J Am Geriatr Soc. 2007;55:189-94.

18. Ivo K, Younsuck K, Ho YY, Sang-Yeon S, Seog HD, Hyunah $\mathrm{B}$, et al. A survey of the perspectives of patients who are seriously ill regarding end-of-life decisions in some medical institutions of Korea, China and Japan. J Med Ethics. 2012;38:310-6. 